\section{Fatores associados ao peso ao nascer de crianças indígenas Terena, residentes na área urbana de Campo Grande, Mato Grosso do Sul, Brasil}

\author{
Factors associated with birthweight of Terena \\ indigenous children living in the urban area of \\ Campo Grande, Mato Grosso do Sul State, Brazil
}

\author{
Factores asociados al peso al nacer de niños \\ indígenas Terena, residentes en el área urbana \\ de Campo Grande, Mato Grosso do Sul, Brasil
}

Deise Bresan 1

Elenir Rose Jardim Cury Pontes 1

Maurício Soares Leite 2

doi: 10.1590/0102-311X00086819

\section{Resumo}

O objetivo do estudo foi verificar a associação do peso ao nascer de crianças Terena, residentes na área urbana de Campo Grande, Mato Grosso do Sul, Brasil, com variáveis demográficas, socioeconômicas e de saúde, e descrever a frequência de baixo peso ao nascer. Os dados foram coletados em visitas domiciliares com formulários aplicados às mães e baseando-se em informações contidas nas Cadernetas da Gestante e da Criança. Foram incluídas todas as mulheres Terena que tiveram filhos nascidos vivos no período de 1o de junho de 2017 a 31 de julho de 2018 e residentes nas aldeias Água Bonita, Darcy Ribeiro, Marçal de Souza e Tarsila do Amaral. O peso ao nascer foi considerado desfecho e as variáveis demográficas, socioeconômicas e de saúde foram consideradas exposição. Participaram do estudo 43 mães e crianças. A frequência de baixo peso ao nascer foi de 2,3\%. No modelo final, as crianças que residiam em domicílios com fossa séptica ou rudimentar apresentaram peso ao nascer menor quando comparadas às que residiam em domicílios com rede coletora de esgoto; e aquelas filhas de mães obesas antes da gestação apresentaram maior peso ao nascer quando comparadas às que nasceram de mães eutróficas segundo o indice de massa corporal pré-gestacional. Os dados buscam trazer à tona discussões sobre as condições de saúde e nutrição dessa importante parcela da população indígena que vive em áreas urbanas.

Índios Sul-Americanos; Saúde de Populações Indígenas; Peso ao Nascer

\author{
Correspondência \\ D. Bresan \\ Rua Dr. Werneck 41, apto. 5303, Campo Grande, MS \\ 79060-300, Brasil. \\ deisebresan@yahoo.com.br \\ 1 Universidade Federal de Mato Grosso do Sul, Campo Grande, \\ Brasil. \\ 2 Universidade Federal de Santa Catarina, Florianópolis, Brasil.
}




\section{Introdução}

No Brasil, segundo dados do Instituto Brasileiro de Geografia e Estatística (IBGE), em um universo de 817.900 indígenas, cerca de $40 \%$ residem em áreas urbanas ${ }^{1}$. Embora representem uma importante parcela da população, a falta de investigações torna o seu perfil de saúde materno-infantil praticamente desconhecido. Além disso, a não inserção dos mesmos na Política Nacional de Atenção à Saúde dos Povos Indígenas 2 contribui ainda mais para a sua invisibilidade frente a contextos locais e regionais de saúde.

Assim, considerando a importância do peso ao nascer como indicador das condições ambientais e de saúde experimentadas durante a gestação e como preditor do crescimento ao longo da infância e de doenças crônicas não transmissíveis na vida adulta 3,4,5,6, o objetivo do estudo foi verificar a associação do peso ao nascer de crianças Terena, residentes na área urbana de Campo Grande, Mato Grosso do Sul, com variáveis demográficas, socioeconômicas e de saúde, e descrever a frequência de baixo peso ao nascer.

\section{Métodos}

Os Terena são a quinta etnia mais numerosa no país (28.845 pessoas), pertencem à família linguística Aruák e os primeiros contatos com não indígenas ocorreram no século XVI 1,7. A partir dos anos de 1930 foram se instalando de maneira dispersa nas periferias de Campo Grande na forma de agrupamentos, dos quais surgiriam, a partir dos anos 1990, loteamentos eminentemente povoados por indígenas 7,8. Não há, contudo, dados atuais e precisos acerca do contingente urbano da população Terena na capital. O presente trabalho foi realizado em quatro loteamentos: Água Bonita, Darcy Ribeiro, Marçal de Souza e Tarsila do Amaral (chamados aqui de aldeias, pois assim eram descritos pelos indígenas).

Foram incluídas no estudo todas as mulheres Terena residentes nas aldeias citadas, que tiveram filhos nascidos vivos no período de 1o de junho de 2017 a 31 de julho de 2018, não sendo realizados processos de amostragem, pois tratou-se de um censo. As entrevistas foram realizadas nos domicílios, com as mulheres. Os dados foram coletados na gestação (dados demográficos e socioeconômicos) e em uma visita até um mês após o nascimento da criança (demais dados). Foram excluídas as crianças nascidas de gestação gemelar e nascidas pré-termo (antes da 37a semana de gestação).

Os dados foram coletados com base em questões no mesmo formato do I Inquérito Nacional de Saúde e Nutrição dos Povos Indígenas ${ }^{9}$, a partir daqui citado como Inquérito Nacional, da Coorte 2015 da Universidade Federal de Pelotas 10, e por meio de exame físico (estatura materna). As Cadernetas de Saúde da Criança e da Gestante foram utilizadas para a obtenção de dados. Os entrevistadores foram treinados e padronizados em antropometria.

A estatura materna foi aferida de acordo com o protocolo de Lohman et al. 11, sendo utilizado antropômetro portátil desmontável Alturexata (Belo Horizonte, Brasil). Usou-se a estatura e o peso pré-gestacional para calcular o índice de massa corporal (IMC) pré-gestacional, classificado segundo a Organização Mundial da Saúde (OMS) 12. O ganho ponderal das gestantes foi classificado segundo o Instituto de Medicina dos Estados Unidos 13. O início do pré-natal foi considerado precoce quando realizado até a 16a semana de gestação ${ }^{14}$. Considerou-se baixo peso ao nascer valores inferiores a $2,500 \mathrm{~kg} 14$

As análises bivariadas e multivariadas foram realizadas por meio de regressão linear, no Stata 11.0 (https://www.stata.com), sendo considerado desfecho o peso ao nascer, que apresentou distribuição normal. Nas análises ajustadas usou-se o processo de seleção para trás (backward). As variáveis independentes foram incluídas segundo determinada hierarquia, em blocos: inicialmente variáveis demográficas e socioeconômicas e posteriormente aquelas relacionadas à saúde materna e ao nascimento da criança. Permaneceram no modelo final aquelas $\operatorname{com} \mathrm{p} \leq 0,20$. Foram consideradas variáveis com significância estatística aquelas que apresentaram valor de $\mathrm{p}<0,05$, segundo teste $\mathrm{F}$.

A pesquisa foi aprovada pelo Comitê de Ética em Pesquisa com Seres Humanos da Universidade Federal de Mato Grosso do Sul (pareceres: 1.992 .298 e 2.252.520) e pela Comissão Nacional de Ética 
em Pesquisa (pareceres: 2.051.925 e 2.295.467). Todas as mães assinaram o Termo de Consentimento Livre e Esclarecido.

\section{Resultados}

De um total de 49 mulheres/crianças elegíveis, participaram do estudo 43 (87,7\%). Houve três recusas, uma desistência e duas mães mudaram de município. Entre as crianças, 2,3\% apresentaram peso < $2,500 \mathrm{~kg}$ e $9,3 \%$ peso igual ou superior a 4,000kg (dados não apresentados em tabelas).

Nas análises bivariadas, as crianças que residiam nas aldeias Marçal de Souza e Tarsila do Amaral apresentaram peso ao nascer maior quando comparadas às que nasceram na Água Bonita $(\mathrm{p}=0,019)$. As crianças que residiam em domicílios com fossa rudimentar ou fossa séptica registraram peso ao nascer menor quando comparadas às que residiam em domicílios com rede coletora de esgoto ( $\mathrm{p}=0,046)$. O peso ao nascer de crianças filhas de mães obesas antes da gestação foi em média $0,440 \mathrm{~kg}$ maior quando comparado ao peso das crianças nascidas de mulheres eutróficas antes da gestação $(\mathrm{p}=0,004)$. As crianças nascidas por parto cesárea eram em média 0,350kg maiores do que as nascidas por parto vaginal $(\mathrm{p}=0,007)$ (Tabela 1$)$.

No modelo final, permaneceram associadas ao peso ao nascer o destino dos dejetos do banheiro e o IMC pré-gestacional (Tabela 2).

\section{Tabela 1}

Coeficiente linear bruto e médias de peso ao nascer de crianças Terena segundo variáveis demográficas, socioeconômicas e de saúde. Campo Grande, Mato Grosso do Sul, Brasil, 2017-2018.

\begin{tabular}{|c|c|c|c|}
\hline \multirow[t]{2}{*}{ Variáveis } & \multirow[t]{2}{*}{$n$ * $(\%)$} & \multicolumn{2}{|c|}{ Peso ao nascer $(\mathbf{k g})$} \\
\hline & & Média (DP) & $\beta$ (IC95\%) \\
\hline Idade (anos) & & & $p=0,736$ \\
\hline $14-22$ & $26(60,5)$ & $3,34( \pm 0,43)$ & 0,00 \\
\hline $23-29$ & $11(25,6)$ & $3,45( \pm 0,39)$ & $0,11(-0,21 ; 0,43)$ \\
\hline$\geq 30$ & $6(13,9)$ & $3,44( \pm 0,57)$ & $0,10(-0,30 ; 0,51)$ \\
\hline Escolaridade (anos) & & & $p=0,402$ \\
\hline $5-8$ & $20(46,5)$ & $3,44( \pm 0,41)$ & 0,00 \\
\hline $9-11$ & $23(53,5)$ & $3,33( \pm 0,46)$ & $-0,11(-0,38 ; 0,15)$ \\
\hline Tempo (anos) que saiu da Terra Indígena & & & $p=0,886$ \\
\hline $1-10$ & $14(32,6)$ & $3,33( \pm 0,39)$ & 0,00 \\
\hline$\geq 11$ & $14(32,6)$ & $3,41( \pm 0,49)$ & $0,07(-0,26 ; 0,41)$ \\
\hline Nasceu na área urbana & $15(34,8)$ & $3,40( \pm 0,43)$ & $0,06(-0,27 ; 0,39)$ \\
\hline Situação conjugal & & & $p=0,467$ \\
\hline Não reside com companheiro & $9(20,9)$ & $3,48( \pm 0,36)$ & 0,00 \\
\hline Reside com companheiro & $34(79,1)$ & $3,36( \pm 0,45)$ & $-0,12(-0,45 ; 0,21)$ \\
\hline Localização do domicílio & & & $p=0,019$ \\
\hline Água Bonita & $12(27,9)$ & $3,13( \pm 0,28)$ & 0,00 \\
\hline Darcy Ribeiro & $9(20,9)$ & $3,26( \pm 0,27)$ & $0,13(-0,22 ; 0,49)$ \\
\hline Marçal de Souza & $16(37,2)$ & $3,53( \pm 0,46)$ & $0,40(0,09 ; 0,70)$ \\
\hline Tarsila do Amaral & $6(14,0)$ & $3,68( \pm 0,56)$ & $0,55(0,14 ; 0,96)$ \\
\hline Número de moradores no domicílio & & & $p=0,553 * *$ \\
\hline $1-3$ & $4(9,3)$ & $3,47( \pm 0,83)$ & 0,00 \\
\hline $4-6$ & $24(55,8)$ & $3,40( \pm 0,39)$ & $-0,73(-0,56 ; 0,41)$ \\
\hline $7-9$ & $15(34,9)$ & $3,33( \pm 0,40)$ & $-0,13(-0,64 ; 0,37)$ \\
\hline
\end{tabular}

(continua) 
Tabela 1 (continuação)

\begin{tabular}{|c|c|c|c|}
\hline \multirow[t]{2}{*}{ Variáveis } & \multirow[t]{2}{*}{$n *(\%)$} & \multicolumn{2}{|c|}{ Peso ao nascer (kg) } \\
\hline & & Média (DP) & $\beta(I C 95 \%)$ \\
\hline Renda per capita $(\mathrm{R} \$)$ & & & $p=0,980$ \\
\hline 1o tercil $(120,00-184,99)$ & $14(35,0)$ & $3,42( \pm 0,37)$ & 0,00 \\
\hline 2o tercil $(185,00-265,99)$ & $12(30,0)$ & $3,38( \pm 0,41)$ & $-0,03(-0,38 ; 0,31)$ \\
\hline 3o tercil $(266,00-800,00)$ & $14(35,0)$ & $3,40( \pm 0,50)$ & $-0,01(-0,35 ; 3,19)$ \\
\hline Destino dos dejetos do banheiro & & & $p=0,046$ \\
\hline Rede coletora & $11(25,6)$ & $3,64( \pm 0,46)$ & 0,00 \\
\hline Fossa séptica & $28(65,1)$ & $3,32( \pm 0,413)$ & $-0,32(-0,62 ;-0,02)$ \\
\hline Fossa rudimentar/rasa & $4(9,3)$ & $3,12( \pm 0,22)$ & $-0,52(-1,01 ;-0,03)$ \\
\hline Início do pré-natal & & & $p=0,645$ \\
\hline Precoce (até a 16a semana) & $25(58,1)$ & $3,41( \pm 0,44)$ & 0,00 \\
\hline Tardio (após a 16 semana) & $18(41,9)$ & $3,34( \pm 0,43)$ & $-0,06(-0,33 ; 0,21)$ \\
\hline Número de consultas de pré-natal & & & $p=0,806$ \\
\hline $1-3$ & $7(16,3)$ & $3,36( \pm 0,44)$ & 0,00 \\
\hline $4-5$ & $15(34,9)$ & $3,44( \pm 0,40)$ & $0,08(-0,32 ; 0,49)$ \\
\hline$\geq 6$ & $21(48,8)$ & $3,35( \pm 0,47)$ & $-0,01(-0,40 ; 0,37)$ \\
\hline IMC pré-gestacional & & & $p=0,004 * *$ \\
\hline Eutrofia & $16(39,0)$ & $3,22( \pm 0,31)$ & 0,00 \\
\hline Sobrepeso & $13(31,7)$ & $3,37( \pm 0,36)$ & $0,14(-0,14 ; 0,44)$ \\
\hline Obesidade & $12(29,3)$ & $3,67( \pm 0,49)$ & $0,44(0,14 ; 0,75)$ \\
\hline Ganho ponderal & & & $p=0,070 * *$ \\
\hline Insuficiente & $10(28,6)$ & $3,22( \pm 0,34)$ & 0,00 \\
\hline Adequado & $7(20,0)$ & $3,50( \pm 0,48)$ & $0,27(-0,15 ; 0,70)$ \\
\hline Excessivo & $18(51,4)$ & $3,55( \pm 0,44)$ & $0,32(-0,01 ; 0,66)$ \\
\hline Sexo da criança & & & $p=0,127$ \\
\hline Masculino & $22(51,2)$ & $3,28( \pm 0,45)$ & 0,00 \\
\hline Feminino & $21(48,8)$ & $3,49( \pm 0,40)$ & $0,20(-0,06 ; 0,46)$ \\
\hline Ordem de nascimento da criança & & & $p=0,452$ \\
\hline $1^{\circ}$ filho & $17(39,6)$ & $3,28( \pm 0,46)$ & 0,00 \\
\hline $2^{\circ}$ filho & $13(30,2)$ & $3,46( \pm 0,43)$ & $0,18(-0,14 ; 0,50)$ \\
\hline $3^{\circ}$ filho ou mais & $13(30,2)$ & $3,44( \pm 0,40)$ & $0,16(-0,15 ; 0,49)$ \\
\hline Tipo de parto & & & $p=0,007$ \\
\hline Vaginal & $26(60,5)$ & $3,24( \pm 0,30)$ & 0,00 \\
\hline Cesariana & $17(39,5)$ & $3,60( \pm 0,52)$ & $0,35(0,09 ; 0,60)$ \\
\hline
\end{tabular}

ß: coeficiente linear; DP: desvio padrão; IC95\%: intervalo de 95\% de confiança; IMC: índice de massa corporal. Nota: coeficientes lineares destacados em negrito apresentam diferença estatística significativa $(p<0,05)$.

* $\mathrm{n}$ máximo para cada categoria, que pode ser diferente entre as variáveis devido a dados ausentes.

** Teste para tendência linear.

\section{Discussão}

A frequência de baixo peso ao nascer registrada entre os Terena aqui avaliados foi de 2,3\%, três vezes menor do que a frequência registrada no Inquérito Nacional ${ }^{9}$. No entanto, nosso estudo incluiu apenas crianças a termo, sendo excluída uma criança nascida pré-termo, o que pode explicar em partes a diferença encontrada. Além disso, os indicadores de qualidade do pré-natal, registrados entre os Terena, delineiam um quadro mais favorável do que aquele que emerge do Inquérito Nacional 9. Na comparação, o pré-natal foi universalmente realizado entre as mulheres Terena, que também registraram média de consultas superior ${ }^{9}$, embora nos dois casos os indicadores estão aquém das recomendações oficiais 14 e destoam negativamente do cenário nacional não indígena 15 . Uma revisão 
Tabela 2

Coeficiente linear ajustado * do peso ao nascer de crianças Terena segundo variáveis independentes. Campo Grande, Mato Grosso do Sul, Brasil, 2017-2018.

\begin{tabular}{|c|c|c|c|}
\hline Variáveis & $\mathbf{n} * *$ & $\begin{array}{c}\text { Peso ao nascer (kg) } \\
\beta(\mathrm{IC} 95 \%)\end{array}$ & Valor de p \\
\hline Destino dos dejetos do banheiro & & $p=0,036$ & \\
\hline Rede coletora & 11 & 0,00 & \\
\hline Fossa séptica & 28 & $-0,33(-0,63 ;-0,03)$ & 0,028 \\
\hline Fossa rudimentar/rasa & 4 & $-0,56(-1,05 ;-0,06)$ & 0,028 \\
\hline IMC pré-gestacional & & $\mathrm{p}=0,030 * \star \star$ & \\
\hline Eutrofia & 16 & 0,00 & \\
\hline Sobrepeso & 13 & $0,13(-0,16 ; 0,42)$ & 0,374 \\
\hline Obesidade & 12 & $0,35(0,03 ; 0,67)$ & 0,031 \\
\hline
\end{tabular}

ß: coeficiente linear; IC95\%: intervalo de 95\% de confiança; IMC: índice de massa corporal.

* Ajustado para outras variáveis do mesmo nível ou de nível superior com valor de $p \leq 0,20$. Permaneceram no modelo

reduzido final apenas as variáveis com valor de $p \leq 0,20$, que estão apresentadas nesta tabela;

** n máximo para cada categoria, que pode ser diferente entre as variáveis devido a dados ausentes;

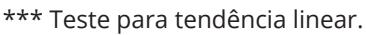

sistemática da literatura revelou que o acesso limitado aos serviços de saúde e a localização das aldeias em áreas rurais ou remotas foram fatores de risco para o baixo peso ao nascer entre povos nativos 3 .

Além disso, as condições sanitárias podem ter um papel importante na gênese dessas diferenças, na medida em que as mães e crianças aqui avaliadas se encontram em um contexto urbano, onde as condições de saneamento são usualmente melhores que as de contextos rurais indígenas ${ }^{9}$ - ainda que distantes do ideal. Entre os Terena, após análise ajustada, o peso ao nascer foi associado ao destino dos dejetos do banheiro, apontando para o impacto das condições sanitárias sobre o peso ao nascer. Condições ambientais e socioeconômicas desfavoráveis também estão associadas a maiores frequências de baixo peso ao nascer 3,4 .

O modelo final também indicou a associação do peso ao nascer com o IMC pré-gestacional: mulheres obesas tiveram crianças com peso maior quando comparadas às eutróficas. Se a associação explica em parte a relativamente baixa frequência de baixo peso ao nascer, ao mesmo tempo indica um quadro de inadequação das condições de nutrição materna, o que pode levar a alterações nos padrões de crescimento físico das crianças durante a gestação e após o nascimento, além da ocorrência de doenças crônicas não transmissíveis na vida adulta 5,6. No contexto Terena a associação ganha importância diante da elevada prevalência de excesso de peso registrada. A frequência de obesidade é o dobro daquela observada entre as mulheres indígenas avaliadas no Inquérito Nacional 9. Sabe-se que a urbanização pode acelerar as mudanças nos padrões alimentares e de atividade física de povos nativos, as quais favorecem o surgimento de sobrepeso e obesidade 16 .

Assim, os dados aqui apresentados buscam trazer à tona discussões relacionadas às condições de saúde e nutrição dessa importante parcela da população indígena que vive em contextos urbanos, até o momento raramente investigados, com vistas à redução de desfechos desfavoráveis para mães e bebês e à reversão das desigualdades registradas. 


\section{Colaboradores}

D. Bresan participou da concepção do projeto, análise e interpretação dos dados, redação do manuscrito e aprovação final da versão a ser publicada. E. R. J. C. Pontes e M. S. Leite participaram da concepção do projeto, análise e interpretação dos dados, revisão crítica relevante do conteúdo intelectual e aprovação final da versão a ser publicada.

\section{Informações adicionais}

ORCID: Deise Bresan (0000-0003-3984-5919); Elenir Rose Jardim Cury Pontes (0000-0003-27110667); Maurício Soares Leite (0000-0002-4183375X).

\section{Agradecimentos}

À Fundação de Apoio ao Desenvolvimento do Ensino, Ciência e Tecnologia do Estado de Mato Grosso do Sul (FUNDECT) e ao Departamento de Ciência e Tecnologia, Secretaria de Ciência, Tecnologia e Insumos Estratégicos, Ministério da Saúde (Decit/ SCTIE/MS), por intermédio do Conselho Nacional de Desenvolvimento Científico e Tecnológico (CNPq), pelo financiamento da pesquisa.

\section{Referências}

1. Instituto Brasileiro de Geografia e Estatística. Censo demográfico 2010: características gerais dos indígenas. Rio de Janeiro: Instituto Brasileiro de Geografia e Estatística; 2012.

2. Ministério da Saúde. Política Nacional de Atenção à Saúde dos Povos Indígenas. Brasília: Ministério da Saúde; 2002.

3. Barreto CTG, Tavares FG, Theme-Filha M, Cardoso AM. Factors associated with low birth weight in indigenous populations: a systematic review of the world literature. Rev Bras Saúde Mater Infant 2019; 19:7-23.

4. Sadovsky AD, Matijasevich A, Santos IS, Barros FC, Miranda AE, Silveira MF. LBW and IUGR temporal trend in 4 population-based birth cohorts: the role of economic inequality. BMC Pediatr 2016; 16:115.

5. Barker DJP. Fetal and infant origins of adult disease. BMJ 1990; 301:1111.

6. Cameron N. Growth patterns in adverse environments. Am J Hum Biol 2007; 19:615-21.

7. Oliveira RC. Urbanização e tribalismo. Rio de Janeiro: Zahar; 1968.

8. Mussi VPL. As estratégias de inserção dos índios Terena: da aldeia ao espaço urbano (19902005) [Tese de Doutorado]. Assis: Faculdade de Ciências e Letras de Assis, Universidade Estadual Paulista; 2006.

9. Associação Brasileira de Saúde Coletiva. Inquérito Nacional de Saúde e Nutrição dos Povos Indígenas: relatório final. Rio de Janeiro: Associação Brasileira de Saúde Coletiva; 2009.

10. Universidade Federal de Pelotas. Questionários e instrumentos utilizados na Coorte 2015. http://www.epidemio-ufpel.org.br/site/con tent/coorte_2015/questionarios.php (acessado em 03/Nov/2015).

11. Lohman TG, Roche AF, Martorell R. Anthropometric standardization reference manual. Champaign: Human Kinetics; 1988.

12. World Health Organization. Obesity: preventing and managing the global epidemic. Geneva: World Health Organization; 1997.

13. Rasmussen KM, Yaktine AL, editors. Weight gain during pregnancy: reexamining the guidelines. Washington DC: National Academies Press; 2009.

14. Ministério da Saúde. Atenção ao pré-natal de baixo risco. Brasília: Ministério da Saúde; 2013.

15. Viellas EF, Domingues RMSM, Dias MAB, Gama SGN, Theme-Filha MM, Costa JV, et al. Assistência pré-natal no Brasil. Cad Saúde Pública 2014; 30 Suppl:S85-S100.

16. Gracey $M$, King $M$. Indigenous health part 1: determinants and disease patterns. Lancet 2009; 374:65-75. 


\section{Abstract}

The study aimed to verify the association between birthweight of Terena children living in the urban area of Campo Grande, Mato Grosso do Sul State, Brazil, and demographic, socioeconomic, and health variables and to describe the prevalence of low birthweight. Data were collective through home visits with a form completed with the mothers and based on records from the prenatal and neonatal cards. The sample included all the Terena women who gave birth to liveborn children from June 1, 2017, to July 31, 2018, and living in the Água Bonita, Darcy Ribeiro, Marçal de Souza, and Tarsila do Amaral villages. Birthweight was the outcome, and demographic, socioeconomic, and health characteristics were the exposure variables. A total of 43 mothers participated with their children. Low birthweight prevalence was $2.3 \%$. In the final model, children living in households with makeshift cesspools showed lower birthweight when compared to those living in households connected to the public sewage disposal system, and daughters of mothers who were obese prior to the pregnancy presented higher birthweight than those born to normal weight mothers, according to pregestational body mass index. The data seek to contribute to discussions on the health and nutrition of this important share of the indigenous population that lives in urban areas.

South American Indians; Health of Indigenous Peoples; Birth Weight

\section{Resumen}

El objetivo del estudio fue verificar la asociación del peso al nacer de niños Terena, residentes en el área urbana de Campo Grande, Mato Grosso do Sul, Brasil, con variables demográficas, socioeconómicas y de salud, así como describir la frecuencia de bajo peso al nacer. Los datos se recogieron en visitas domiciliarias con formularios aplicados a las madres, y a partir de información que contenían los Cuadernillos de la Gestante y del Niño. Se incluyeron a todas las mujeres Terena que tuvieron hijos nacidos vivos, durante el período del 1 de junio de 2017 a 31 de julio de 2018, y residentes en las aldeas Água Bonita, Darcy Ribeiro, Marçal de Souza y Tarsila do Amaral. El peso al nacer se consideró un resultado y las variables demográficas, socioeconómicas y de salud se consideraron exposición. Participaron en el estudio 43 madres y niños. La frecuencia de bajo peso al nacer fue de un 2,3\%. En el modelo final, los niños que residían en domicilios con fosa séptica o rudimentaria presentaron un peso al nacer menor, cuando se comparan con los que residian en domicilios con una red de alcantarillado; $y$ aquellos hijos de madres obesas antes de la gestación presentaron un mayor peso al nacer, cuando se comparan con los que nacieron de madres eutróficas, según el índice de masa corporal pregestacional. Los datos pretenden plantear discusiones sobre condiciones de salud $y$ nutrición de esta importante parte de la población indígena que vive en áreas urbanas.

Indios Sudamericanos; Salud de Poblaciones Indígenas; Peso al Nacer
Recebido em 07/Mai/2019

Versão final reapresentada em 08/Ago/2019

Aprovado em 09/Ago/2019 
Bresan D, Pontes ERJC, Leite MS. Fatores associados ao peso ao nascer de crianças indígenas Terena, residentes na área urbana de Campo Grande, Mato Grosso do Sul, Brasil. Cad Saúde Pública 2019; 35 Suppl 3:e00086819.

doi: 10.1590/0102-311X00086819

Onde se lê:

\section{Agradecimentos}

À Fundação de Apoio ao Desenvolvimento do Ensino, Ciência e Tecnologia do Estado de Mato Grosso do Sul (FUNDECT), pelo financiamento da pesquisa.

Leia-se:

\section{Agradecimentos}

À Fundação de Apoio ao Desenvolvimento do Ensino, Ciência e Tecnologia do Estado de Mato Grosso do Sul (FUNDECT) e ao Departamento de Ciência e Tecnologia, Secretaria de Ciência, Tecnologia e Insumos Estratégicos, Ministério da Saúde (Decit/SCTIE/MS), por intermédio do Conselho Nacional de Desenvolvimento Científico e Tecnológico (CNPq), pelo financiamento da pesquisa. 